\title{
Crecimiento compensatorio y producción en las fases de precría, preengorde y engorde comercial del camarón blanco, Litopenaeus vannamei, en Costa Rica
}

\author{
Compensatory growth and production of white shrimp, \\ Litopenaeus vannamei, in Costa Rica, during the maternity, \\ development and grow-out commercial phases
}

\author{
José A. Valverde-Moya ${ }^{1}$ y Jorge Alfaro-Montoya ${ }^{2 *}$
}

\begin{abstract}
RESUMEN
Este estudio evaluó el crecimiento compensatorio y la producción de Litopenaeus vannamei en cultivos comerciales, durante el 2014, en fincas del Golfo de Nicoya, Costa Rica. Se compararon el crecimiento y la producción en tres sistemas a partir de una fase de precría (PC) con alta densidad de siembra directa de poslarvas $\left(85 / \mathrm{m}^{2}\right)$ : a) precría-preengorde (PC-PE) en densidad media $\left(23.3 / \mathrm{m}^{2}\right)$ y peso inicial de $1.4 \mathrm{~g}, \mathrm{~b}$ ) precría-preengorde-engorde (PC-PE-E) en baja densidad $\left(8.6 / \mathrm{m}^{2}\right)$ y peso inicial de $6.0 \mathrm{~g}$ y c) precría-engorde (PC-E) $\left(9.4 / \mathrm{m}^{2}\right.$ y $\left.1.4 \mathrm{~g}\right)$. Se registró un aumento en el crecimiento en la PC-PE ( $0.85 \mathrm{~g} / \mathrm{semana})$ con respecto a la PC $(0.26 \mathrm{~g} / \mathrm{semana})$ y la tasa específica de crecimiento (TEC) fue de $3.73 \% \mathrm{~g} / \mathrm{d}$ en la PC-PE. La sobrevivencia $(81.0 \%)$ y la producción (1 $029 \mathrm{~kg} / \mathrm{ha})$ en la PC-PE fueron superiores con respecto a la PC (75.3\% y $842 \mathrm{~kg} /$ ha, respectivamente). El crecimiento semanal no mostró diferencias entre ambos sistemas de engorde: $\mathrm{PC}-\mathrm{E}=1.07$ y PC-PE-E $=1.02 \mathrm{~g} / \mathrm{semana}$; sin embargo, la TEC fue inferior en la PC-PE-E (1.51\%g/d) con respecto a la PC-E $(3.19 \% \mathrm{~g} / \mathrm{d})$. Los resultados indican la inexistencia de un crecimiento compensatorio entre PC y PC-PE, así como entre PC-PE y PC-PE-E. Crecimiento compensatorio parcial se registró entre $\mathrm{PC}$ y $\mathrm{PC}$-E. Tomando en cuenta la ganancia compensatoria en el crecimiento de los juveniles, el cultivo de $L$. vannamei en PC a alta densidad parece ser una buena estrategia de producción.
\end{abstract}

Palabras claves: Decapoda, Penaeidae, Litopenaeus vannamei, cultivo comercial, camarones.

\begin{abstract}
Compensatory growth and production of Litopenaeus vannamei in commercial cultures in farms in the Gulf of Nicoya, Costa Rica, during 2014 were evaluated in this study. Growth and production were compared between three culture systems starting from an initial maternity (MA) phase with high stocking density $\left(85 / \mathrm{m}^{2}\right)$ of post larvae: a) maternity-development (MA-DE) with a medium stocking density $\left(23.3 / \mathrm{m}^{2}\right)$ and an initial weight of 1.4 $\mathrm{g}, \mathrm{b})$ maternity-development-grow-out (MA-DE-G) with a low density $\left(8.6 / \mathrm{m}^{2}\right)$ and an initial weight of $6.0 \mathrm{~g}$ and c) maternity-grow-out (MA-G) $\left(9.4 / \mathrm{m}^{2}\right.$ and $\left.1.4 \mathrm{~g}\right)$. An increase in growth was recorded in MA-DE $(0.85 \mathrm{~g} /$ week $)$ as compared to MA $(0.26 \mathrm{~g} / \mathrm{week})$, and the specific growth rate (SGR) was $3.73 \% \mathrm{~g} /$ day for MA-DE. Survival $(81.0 \%)$ and production (1 $029 \mathrm{~kg} / \mathrm{ha})$ in MA-DE were higher than in MA $(75.3 \%$ and $842 \mathrm{~kg} /$ ha, respectively). Weekly growth showed no differences between both grow-out systems: $M A-G=1.07$ and MA-DE-G $=1.02 \mathrm{~g} /$ week; however, SGR was lower in MA-DE-G (1.51\%g/day) than in MA-G (3.19\%g/day). Results indicate the lack of compensatory growth from MA to MA-DE, and from MA-DE to MA-DE-G, while partial compensatory growth was recorded from MA to MA-G. Taken into account this compensatory growth gain in juveniles, the culture of $L$. vannamei in MA at high density seems to be a good production strategy.
\end{abstract}

Keywords: Decapoda, Penaeidae, Litopenaeus vannamei, commercial culture, shrimp.

\footnotetext{
Consultor en Acuicultura, jvalmo@yahoo.com

2 Escuela de Ciencias Biológicas, Universidad Nacional, Heredia, Costa Rica, jorge.alfaro.montoya@una.cr*
}

Recibido: 30 de marzo de 2015

Corregido: 1 de junio de 2015

Aceptado: 26 de julio de 2015

DOI: http://dx.doi.org/10.15359/revmar.7.7

Rev. Mar. Cost. ISSN 1659-455X. Vol. 7: 99-115, Diciembre 2015. 


\section{INTRODUCCIÓN}

La productividad y rentabilidad económica del cultivo comercial de camarones marinos en el Golfo de Nicoya, Costa Rica, ha sido evaluada y se determinó que fue mayor en tres ciclos cortos de cultivo (90-120 días) con respecto a un solo ciclo largo (180 días) (Valverde-Moya \& AlfaroMontoya, 2014). Sin embargo, se indica que la estrategia de producción no debe ser estricta sino flexible para ajustarse sobre todo a las variaciones del precio tanto nacional como internacional, en función de las tallas y duración de ciclos más rentables.

El número de ciclos cortos se ha tratado de incrementar en algunas fincas camaroneras del Golfo de Nicoya con la implementación del cultivo en fases a alta densidad en estanques de maternidad o precría para pasarlos después a estanques de desarrollo o preengorde a mediana densidad y de engorde a baja densidad tradicionalmente utilizada hasta la talla comercial. Una estrategia similar de producción fue común en la década de mil novecientos ochenta en otros países como Brasil, pero ha ido decayendo con el paso de los años debido a varias razones como son: el elevado costo en la construcción de estanques pequeños, la ocupación de gran cantidad de área que de otra forma se hubiera utilizado para la etapa de engorde, la extremadamente laboriosa cosecha y traslado de los juveniles a los estanques más grandes y el riesgo alto de esta labor debido al estrés infligido a los animales por su manipulación, pesaje y transferencia (Nunes, 2011).
Esta estrategia de cultivo se justifica solo si demuestra ser más eficiente y productiva que la manera tradicional y convencional como lo es la siembra directa en los estanques de producción. Una forma de acortar los ciclos de cultivo incrementando la producción sería por medio del crecimiento compensatorio del camarón al pasarlo de etapas o fases en alta densidad a otras menos densas y con mayor disponibilidad de espacio y alimento.

El crecimiento compensatorio ha sido definido por muchos autores como una fase de crecimiento rápido cuando las condiciones favorables han sido restablecidas después de un periodo de restricciones sin llegar a afectar la integridad física de manera letal o subletal (Delgado-Vidal et al. 2009). Las restricciones comunes son: privaciones de alimento, bajas temperaturas, depleciones de oxígeno disuelto y contaminación del agua, entre otras (Stumpf et al. 2009; Marques \& Lombardi, 2011). Según los mismos autores, el crecimiento puede manifestarse en diferentes formas: sobrecompensación, compensación total, compensación parcial o no compensación, dependiendo de diferentes factores como la especie, la competencia, la variación ambiental, la alimentación y otros. Para inducir este proceso se requieren dos fases: una de estrés cuando el crecimiento es reprimido y otra, seguida inmediatamente, cuando desaparecen los depresores del crecimiento y dejan que se restablezca este, pero a una tasa más baja de lo que tiene el potencial fisiológico del animal.

En sistemas de maternidad o precría de camarón en densidad alta se 
recomienda una alimentación continua cada 2 horas, 12 veces al día (Cervais \& Zeigler, 2013). Estas normas son difíciles de cumplir en operaciones comerciales, por lo que es común encontrar que los camarones no exhiben el mismo crecimiento ni llegan a alcanzar la talla de los sembrados directamente en condiciones similares de cultivo en ese periodo de tiempo. Sin embargo, podrían llegar a recuperar rápidamente esa diferencia por medio del crecimiento compensatorio si se le dan las condiciones apropiadas de manejo después de transferirlos a estanques más grandes de desarrollo o engorde definitivo a menor densidad. La evaluación de la compensación del crecimiento sobre la productividad de los cultivos de camarones marinos comerciales requiere de estudios aplicados (Fraga et al. 2003).

Por lo tanto, el primer objetivo de este estudio fue determinar la existencia de crecimiento compensatorio en Litopenaeus vannamei después de un periodo de restricción en la fase de maternidad a densidades relativamente altas, seguida por la fase de desarrollo a densidad mediana y de engorde a densidades bajas comerciales. El segundo objetivo fue determinar sus efectos sobre la producción del cultivo de $L$. vannamei en el Golfo de Nicoya, Costa Rica.

\section{MATERIALES Y MÉTODOS}

El estudio se llevó a cabo desde mediados de febrero hasta inicios de noviembre del 2014 en cuatro fincas comerciales denominadas Palito (12.9 ha), Rocha (4.8 ha), Pajua (19.9 ha) y Pochote (11.8 ha) para un total de 49.4 ha. Las fincas están localizadas en el sector de Corozal de Jicaral perteneciente al distrito de Lepanto, Puntarenas, en la región del Pacífico Norte de Costa Rica, al margen oeste del Golfo de Nicoya y cercanas al estero

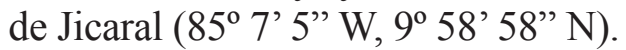

Se sembraron estanques pequeños de maternidad o precría (PC) de 0.5 a 1.0 ha distribuidos en las cuatro fincas en tres ciclos de producción, para un total de 4.6 ha (ciclo I), 6.4 ha (ciclo II) y 6.9 ha (ciclo III). Las siembras se hicieron del 17 al 19 de febrero en el ciclo I, del 15 de abril al 29 de mayo en el ciclo II y del 10 de junio al 23 de septiembre en el ciclo III con poslarvas (PL) recién traídas del laboratorio. Las poslarvas fueron de la misma procedencia (Empresa Activos de Crustáceos, Punta Morales, Costa Rica) y edad (PL 12; $300 \mathrm{PL} / \mathrm{g} ; 3.3 \mathrm{mg} / \mathrm{PL}$ ). Los nauplios para el levantamiento de las larvas y la obtención de las poslarvas en este laboratorio fueron importados de la empresa Biogemar ${ }^{\circledR}$ (Ecuador), bajo protocolos de selección genética. El promedio de las densidades de siembra fue de $85.0 \pm 3.0 / \mathrm{m}^{2}$ dentro de un rango de 81.7 a $87.7 / \mathrm{m}^{2}$ entre ciclos.

En la fase de desarrollo o preengorde (PE) se sembraron juveniles provenientes de la $\mathrm{PC}$ con un peso promedio de $1.4 \pm 0.2 \mathrm{~g}$ en estanques medianos de 0.8 a 1.8 ha distribuidos en tres de las fincas, en tres ciclos, para un total de 9.3 ha (ciclo I), 10.4 ha (ciclo II) y 6.4 ha (ciclo III). Las siembras se hicieron del 18 de febrero al 30 abril en 
el ciclo I, del 15 de mayo al 29 de julio en el ciclo II y entre el 19 de agosto y el 24 de octubre en el ciclo III. El promedio de las densidades de siembra fue de $23.3 \pm 3.2 / \mathrm{m}^{2}$ oscilando de 20.6 a $26.8 / \mathrm{m}^{2}$ entre ciclos.

En la fase de engorde (E) se siguieron dos estrategias:

A) La fase PC-E con juveniles provenientes de la $\mathrm{PC}$ con un peso promedio de $1.4 \pm 0.3 \mathrm{~g}$ en estanques con áreas de $1.0 \mathrm{a} 4.7$ ha distribuidos en tres de las fincas, en tres ciclos, para un total de 15.7 ha (ciclo I), 14.5 ha (ciclo II) y 19.2 ha (ciclo III). Las siembras en esta fase se hicieron del 21 de marzo al 9 de mayo en el ciclo I, del 30 de junio al 7 de julio en el ciclo II y entre el 10 y el 27 de septiembre en el ciclo III. Las densidades de siembra por ciclo oscilaron entre 8.8 y $10.0 / \mathrm{m}^{2}$ con un promedio de $9.4 \pm 0.6 / \mathrm{m}^{2}$.

B) La fase PC-PE-E con juveniles provenientes del preengorde (PC-PE) $\mathrm{y}$ un peso promedio de $6.0 \pm 0.4 \mathrm{~g}$ en estanques de área similar a la anterior en dos ciclos, para un total de 25.2 ha (I ciclo) y 26.8 ha (II ciclo). Las siembras en esta fase se hicieron del 15 de abril al 10 de junio en el ciclo I y del 16 de agosto al 7 de noviembre en el ciclo II. Las densidades de siembra fueron de 7.9 y $9.4 / \mathrm{m}^{2}$ en cada ciclo, respectivamente, con un promedio de $8.6 \pm 1.1 / \mathrm{m}^{2}$.

La siembra en la PC se hizo después de una preparación similar a la de los ciclos cortos siguiendo el plan de manejo convencional descrito previamente (Valverde-Moya \& AlfaroMontoya, 2013). La siembra fue directa en los estanques después de un periodo de aclimatación a la temperatura y salinidad existentes en los estanques en esos momentos. No se hizo evaluación de la sobrevivencia a las 24 y 48 horas después de la siembra.

Los traslados y las siembras de juveniles a las otras fases de desarrollo y engorde se hicieron después de la preparación convencional (ValverdeMoya \& Alfaro-Montoya, 2013), en los estanques una vez a inicios del año. En los ciclos siguientes solo se llenaron y fertilizaron los estanques con urea a razón de $15 \mathrm{~kg} / \mathrm{ha}$ para mantener la turbidez del agua entre 45 y $55 \mathrm{~cm}$ medida con el disco Sechii. Los juveniles se cosecharon por la compuerta de salida con una red fina y se pesaron rápidamente en una balanza marca Ohaus $( \pm 1 \mathrm{~g})$. Se trasladaron por un sistema de bombeo en tubería a los estanques cercanos o en mallas finas dentro de cajas plásticas en motocicleta a estanques más alejados.

Se llevó un registro diario de la temperatura y el oxígeno disuelto en las mañanas y en las tardes y de la turbidez del agua una vez a la semana. La salinidad se midió semanalmente con un refractómetro. Se utilizó aireación de emergencia en las noches con aireador de tractor, con niveles de oxígeno inferior a $3 \mathrm{mg} / \mathrm{l}$. Semanalmente se hicieron muestreos de crecimiento del camarón por parte del biólogo de las fincas según procedimientos establecidos (Valverde-Moya \& Alfaro-Montoya, 2013). Los recambios de agua fueron superficiales y leves $(5 \%)$ en la PC, superficiales y moderados (10\%) en la 
PC-PE y de fondo y fuertes (15-20\%) en las dos fases de engorde hasta el final del cultivo.

La alimentación inicial fue de plancton y detritus en la PC promovida por la fertilización convencional al agua. A partir del tercer día, se aplicó alimento molido (Nicovita ${ }^{\circledR} 35 \%$ de proteína), cuatro veces al día a razón de $5 \mathrm{~kg} /$ ha. Alimento entero ( $35 \%$ proteína) se suministró desde el segundo día en la PC-PE, tres veces al día según la tabla de alimentación del biólogo yla información generada de los comederos (20/ha). En las dos fases de engorde se aplicó alimento con $30 \%$ de proteína desde el inicio, dos veces al día (mañana y tarde) siguiendo la tabla de alimentación y el uso de $100 \%$ de comederos en la misma cantidad. La tabla de alimentación utilizada por el biólogo fue la misma en todos los ensayos, empezando con un $5.0 \%$ del peso promedio a los $2.0 \mathrm{~g}$ y bajando gradualmente hasta terminar con un $2.5 \%$ del peso promedio en la cosecha final.

La demostración de la existencia del crecimiento compensatorio y su efecto sobre la producción del cultivo entre las fases PC y PC-PE se hizo comparando los promedios \pm desviación estándar de sobrevivencia (número final/número inicial *100), crecimiento semanal (peso ganado/ días*7) y conversión alimenticia $(\mathrm{CA}=\mathrm{kg}$ alimento $/ \mathrm{kg}$ ganados de biomasa). Las condiciones experimentales en estas fases que sirvieron de comparación entre ellas son: las respectivas densidades de siembra (alta en la PC $=85.0 \pm 3.0$ / $\mathrm{m}^{2}$ y mediana en la PC-PE $=23.3 \pm$ $3.2 / \mathrm{m}^{2}$ ) y el peso inicial de siembra $(3.3 \mathrm{mg} / \mathrm{PL}$ y juveniles de $1.4 \pm 0.2 \mathrm{~g}$, respectivamente).

Los promedios \pm desviación estándar de los mismos índices (sobrevivencia, crecimiento semanal y conversión alimenticia), además de la producción ( $\mathrm{kg} / \mathrm{ha}$ ), se compararon entre las dos fases de engorde (PC-E vs. PCPE-E). Las condiciones experimentales entre ambas fases que sirvieron de comparación fueron: el peso inicial $(1.35 \pm 0.30 \mathrm{~g}$ en la PC-E y $6.00 \pm 0.42$ $\mathrm{g}$ en la PC-PE-E) y la duración en los ciclos de cultivo, pudiendo realizarse tres ciclos en la PC-E y dos ciclos en la PC-PE-E al año.

El coeficiente de variación (Covar.) para la producción $(\mathrm{kg} / \mathrm{ha})$ se calculó con la fórmula: Covar. = Desvest. / promedio * 100 .

Los indicadores de la producción se obtuvieron de los totales y promedios de las dos fases de engorde. La producción en $\mathrm{kg} / \mathrm{ha} / \mathrm{año}$ se estimó multiplicando el número de ciclos por los $\mathrm{kg} / \mathrm{ha}$ y el rendimiento se calculó con los kilos producidos por cada 1000 juveniles (millar) sembrados.

El peso promedio en la PC se midió $(1.0 \pm 0.5 \mathrm{~g}, \mathrm{n}=30)$ luego del primer mes de cultivo cuando tenían tamaño suficiente para capturarlos y se estimó una tasa de crecimiento de $0.25 \mathrm{~g} / \mathrm{semana}$ hasta alcanzarlo. Al momento de cosecharlos de la PC (36 días de cultivo) para trasladarlos a las otras fases tenían un peso promedio de $1.4 \pm 0.2 \mathrm{~g}$ ( $\mathrm{n}=30$ por estanque). La ganancia en peso de las fases restantes 
se analizó según los pesos medidos cada semana en las visitas del biólogo a las fincas.

La tasa específica de crecimiento (TEC) se obtuvo con la fórmula:

TEC ( $\%$ aumento en peso $(\% \mathrm{~g} /$ día $)=[(\ln \mathrm{Pf}-\ln \mathrm{Pi}) / \mathrm{t}] \times 100$, donde:

$\ln \mathrm{Pf}$ y $\ln \mathrm{Pi}=$ logaritmo natural del peso húmedo final e inicial de cada semana $\mathrm{t}=$ tiempo en días

\section{RESULTADOS}

Las variaciones mensuales de la temperatura y el oxígeno disuelto en las mañanas y en las tardes, así como de la turbidez del agua durante los ciclos de cultivo en el 2014, se muestran en el Cuadro 1. Los valores más bajos de la temperatura se presentaron en las mañanas $\left(27.7 \pm 1.3^{\circ} \mathrm{C}\right)$ y en las tardes $\left(30.5 \pm 1.3^{\circ} \mathrm{C}\right)$ de octubre, mientras que en el resto del año de estudio se mantuvieron más altos y relativamente constantes (de
28.5 a $30.5^{\circ} \mathrm{C}$ en las mañanas y de 31.7 a $33.8^{\circ} \mathrm{C}$ en las tardes).

El oxígeno disuelto presentó los valores más bajos en las mañanas $(1.93 \pm 0.09 \mathrm{mg} / \mathrm{l})$ y en las tardes $(6.43 \pm 0.30 \mathrm{mg} / \mathrm{l})$ del mes de mayo. Valores más altos $(4.76 \pm 0.36 \mathrm{mg} / \mathrm{l} \mathrm{en}$ las mañanas y $8.52 \pm 0.75 \mathrm{mg} / \mathrm{l}$ en las tardes) se presentaron el mes de marzo en plena época seca en esta región del país. En el resto del año fluctuó entre 2.37 y $4.35 \mathrm{mg} / \mathrm{l}$ en las mañanas y de 7.33 a $8.31 \mathrm{mg} / \mathrm{l}$ en las tardes.

El agua fue más clara en el mes de marzo con una turbidez de 51.7 $\pm 4.7 \mathrm{~cm}$, mientras que fue más turbia y no tuvo mayores variaciones $(38.8-45.5 \mathrm{~cm})$ en el resto del año de estudio. La fluctuación semanal de la salinidad durante el estudio coincidió con la mostrada por Valverde-Moya \& Alfaro-Montoya (2013) un año antes, indicando que los ciclos iniciaron con

Cuadro 1. Valores promedio de parámetros fisicoquímicos del agua medidos durante los meses de estudio en todas las fases de cultivo

Table 1. Average physical-chemical parameters of water measured during the study in all the culture phases

\begin{tabular}{lllllllllll}
\hline Meses & \multicolumn{2}{c}{ Disco Sechii $(\mathbf{c m})$} & \multicolumn{3}{c}{ Temperatura $\left({ }^{\circ} \mathbf{C}\right)$} & \multicolumn{3}{c}{ Oxígeno disuelto (mg/l) } \\
& & & \multicolumn{2}{c}{ AM } & \multicolumn{2}{c}{ PM } & \multicolumn{2}{c}{ AM } & PM \\
\hline & Prom. & D. S. & Prom. & D. S. & Prom. & D. S. & Prom. & D. S. & Prom. & D. S. \\
\hline 3 & 51.7 & 4.7 & 29.0 & 0.7 & 32.6 & 0.4 & 4.76 & 0.36 & 8.52 & 0.75 \\
4 & 45.1 & 1.1 & 30.2 & 0.2 & 33.2 & 0.3 & 2.69 & 0.46 & 7.33 & 0.93 \\
5 & 40.6 & 0.7 & 30.5 & 0.3 & 33.1 & 0.2 & 1.93 & 0.09 & 6.43 & 0.30 \\
6 & 41.4 & 1.4 & 30.3 & 0.2 & 33.1 & 0.3 & 2.53 & 0.40 & 7.69 & 0.22 \\
7 & 40.0 & 0.0 & 30.2 & 0.2 & 33.2 & 0.4 & 3.24 & 0.62 & 8.31 & 0.29 \\
8 & 38.8 & 1.7 & 30.3 & 0.0 & 33.8 & 0.2 & 2.37 & 0.07 & 8.30 & 0.21 \\
9 & 40.9 & 2.1 & 29.7 & 0.7 & 32.5 & 0.9 & 2.49 & 0.30 & 8.15 & 0.15 \\
10 & 44.4 & 1.5 & 27.7 & 1.3 & 30.5 & 1.3 & 4.35 & 0.19 & 7.67 & 0.31 \\
11 & 45.5 & 2.4 & 28.5 & 0.5 & 31.7 & 0.5 & 3.66 & 0.34 & 7.77 & 0.44 \\
\hline
\end{tabular}


agua marina ( $35 \mathrm{~g} / \mathrm{l})$ en marzo y que la salinidad se fue reduciendo a partir de mayo hasta llegar a un mínimo de 15 $\mathrm{g} / \mathrm{l}$ en octubre.

Los datos de crecimiento y los rendimientos de producción de los tres ciclos en las fases PC y PC-PE se presentan en el Cuadro 2 con sus valores respectivos (totales y promedios) en el año 2014. Se encontró un aumento en la tasa de sobrevivencia de la PCPE $(81.0 \pm 10.6 \%)$ con respecto a la PC $(75.3 \pm 4.2 \%)$ en mayor densidad de siembra. El crecimiento semanal también fue mayor en la PC-PE $(0.85$ $\pm 0.22 \mathrm{~g} / \mathrm{semana})$ que en la PC $(0.26$ $\pm 0.02 \mathrm{~g} /$ semana). La TEC disminuyó considerablemente en la PC-PE $(3.73 \pm 0.66 \% \mathrm{~g} / \mathrm{d})$ en relación con la PC $(17.01 \pm 0.79 \% \mathrm{~g} / \mathrm{d})$ donde se sembraron poslarvas. La producción fue superior (1 $099 \pm 193 \mathrm{~kg} / \mathrm{ha})$ en la PC-PE que en la PC ( $842 \pm 193 \mathrm{~kg} / \mathrm{ha})$. La conversión alimenticia también fue mayor en la PC-PE (1.60:1.00) que en la PC (0.90:1.00), así como la tasa de alimentación (PC-PE $=25.10 \pm 1.21 \mathrm{y}$ $\mathrm{PC}=19.77 \pm 1.82 \mathrm{~kg}$ alim $/ \mathrm{ha} / \mathrm{d})$.

En el Cuadro 3 se muestran los rendimientos de producción de los ciclos en las fases de engorde (PC-E y PC-PE-E). La principal diferencia entre ellas es el peso inicial de siembra al utilizar juveniles de la fase PC (1.35 $\pm 0.30 \mathrm{~g})$ en la PC-E y de la fase PCPE $(6.00 \pm 0.42 \mathrm{~g})$ en la PC-PE-E. Otra diferencia importante entre ambas fases fue la duración de los ciclos de cultivo. En la fase PC-E la duración total fue de 113 días compuestos por 36 días en la PC y 77 días en la PC-E. Mientras tanto, en la PC-PE-E la duración total fue de 127 días constituidos por 36 días en la PC, 41 días en la PC-PE y 50 días en la PC-PE-E. Debido a ello, se pudieron realizar tres ciclos de cultivo al año en la PC-E y únicamente dos ciclos al año en la PC-PE-E.

Con una densidad de siembra similar $\left(9.4 \pm 0.6 / \mathrm{m}^{2}\right.$ en la PC-E y $8.7 \pm$ $1.1 / \mathrm{m}^{2}$ en la PC-PE-E), se obtuvieron en ambas fases resultados muy parecidos de sobrevivencia $(84.3 \pm 11.2$ y $85.5 \pm$ $7.8 \%)$, peso final a la cosecha (12.8 \pm 0.5 y $12.5 \pm 1.0 \mathrm{~g}$ ), crecimiento semanal $(1.07 \pm 0.13$ y $1.02 \pm 0.35 \mathrm{~g} / \mathrm{semana}) \mathrm{y}$ producción $(995 \pm 152$ y $910 \pm 141 \mathrm{~kg} /$ ha) de manera respectiva en cada fase. El coeficiente de variación fue del 15\% en ambas fases.

Las mayores diferencias entre ambas fases se presentaron en la biomasa ganada, la conversión alimenticia y la TEC. En el primer caso fue casi el doble en la PC-E (41 $984 \mathrm{~kg}$ ) que en la PC-PE-E (24 $795 \mathrm{~kg}$ ) debido a la realización de un ciclo más de cultivo al año en esta fase. La conversión alimenticia fue considerablemente más baja en la fase PC-E (1.40 $\pm 0.17: 1.00)$ que en la PC-PE-E (2.05 $\pm 0.64: 1.00)$. Finalmente, la TEC fue superior en la fase PC-E $(3.19 \pm 0.36 \% \mathrm{~g} / \mathrm{d})$ que en la PC-PE-E $(1.51 \pm 0.6 \% \mathrm{~g} / \mathrm{d})$. También fue superior en la fase anterior (PCPE: $3.73 \pm 0.66 \% \mathrm{~g} / \mathrm{d}$ ) a la PC-PE-E.

Al realizar tres ciclos al año en la PC-E y dos ciclos anuales en la PC-PE-E, la producción fue considerablemente superior $(995 \mathrm{~kg} / \mathrm{ha} * 3=2985 \mathrm{~kg} / \mathrm{ha} / \mathrm{año})$ en la primera que en la segunda $(910 \mathrm{~kg} /$ ha $* 2=1820 \mathrm{~kg} / \mathrm{ha} / \mathrm{año})$. No obstante, 


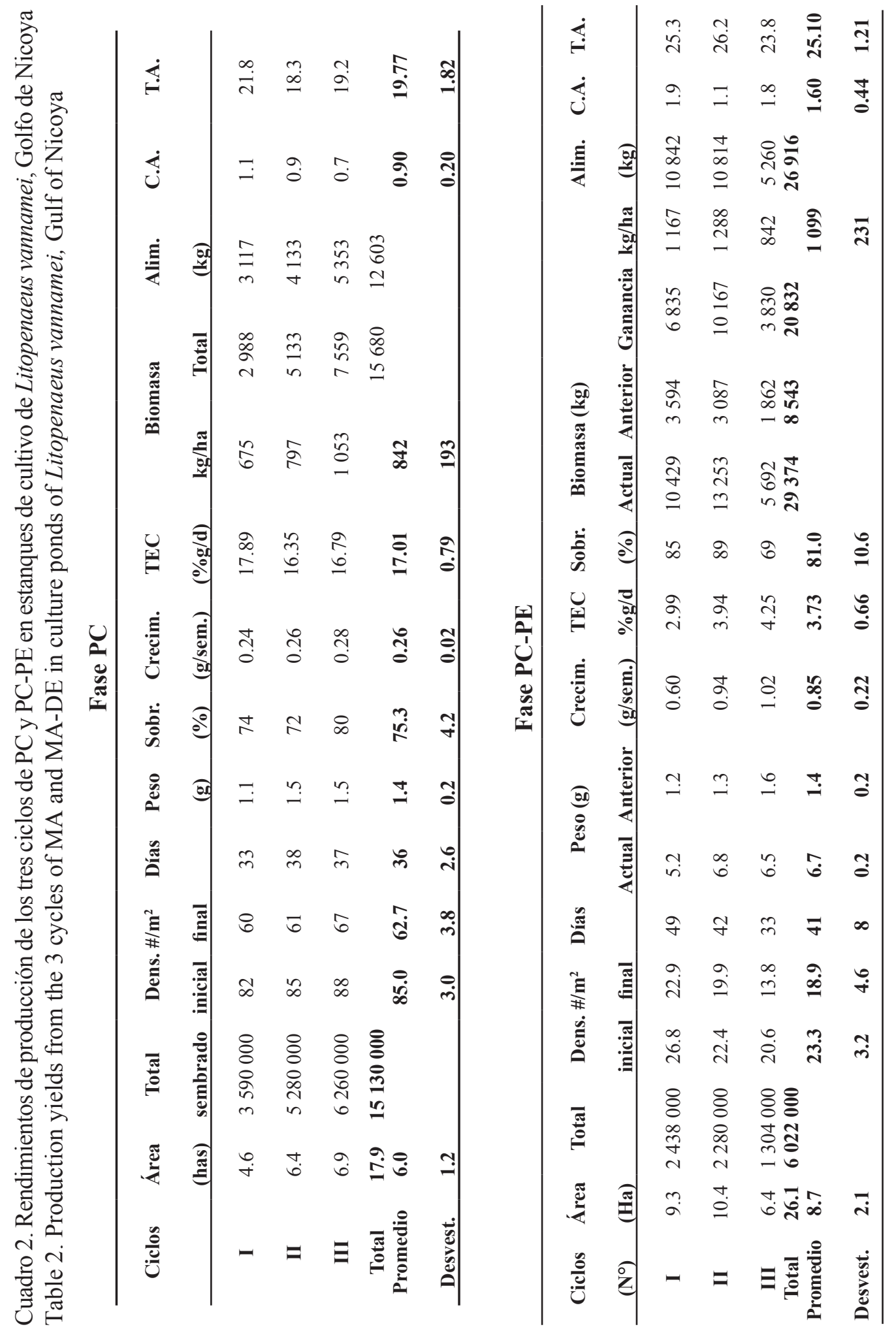


$\frac{0}{\circ}$

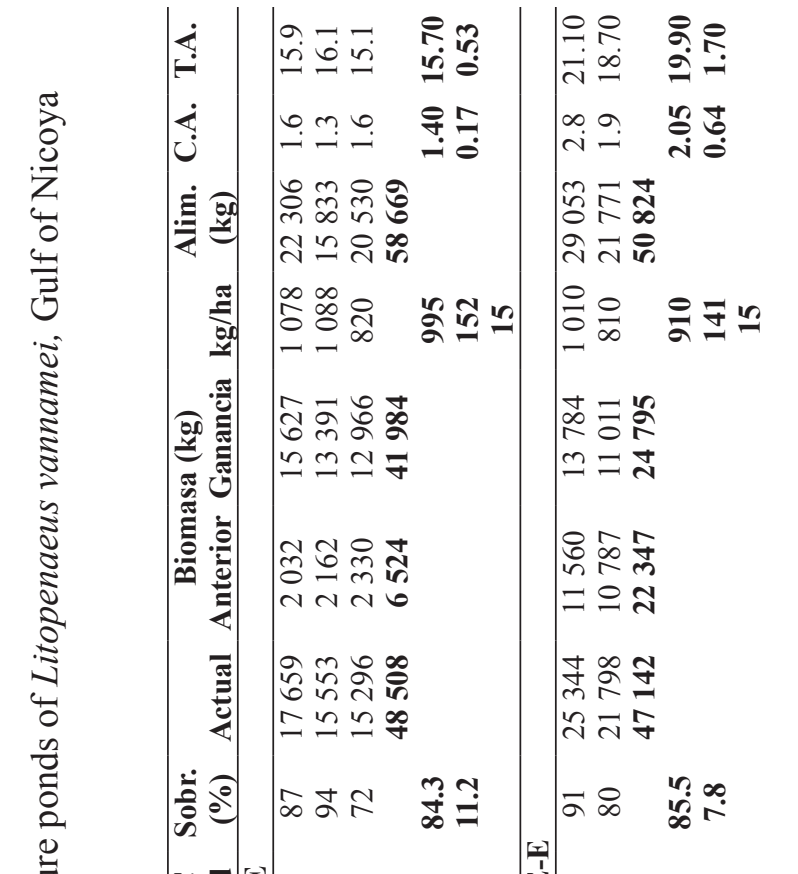

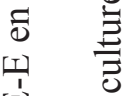

II

品

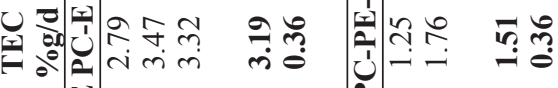

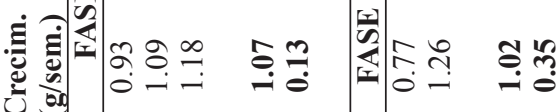

2

in

$\sum$

ช

$\frac{0}{0}$

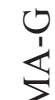

20

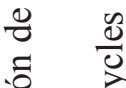

恣

कo

焉

‡

o

on

$\dot{0} \pi$

○ें

䒿 $\frac{0}{0} \frac{0}{0}$

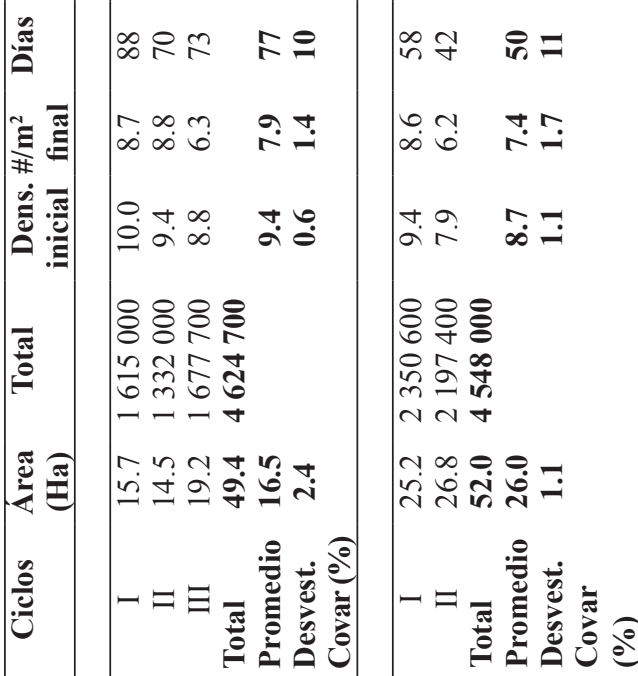


el rendimiento medido como la cantidad de kilos producidos por cada millar de juveniles sembrados fue similar en ambas fases. En la PC-E fue de 10.49 $\mathrm{kg} /$ millar y en la PC-PE-E fue de 10.37 $\mathrm{kg} /$ millar.

El aumento en el peso medido semanalmente en todas las fases de estudio se muestra en la Figura 1. El peso final de la fase $\mathrm{PC}(1.37 \pm 0.23 \mathrm{~g})$ con alta densidad de siembra mejoró en la segunda semana después de que los juveniles se trasladaron a las fases PC-PE $(2.61 \pm 1.22 \mathrm{~g})$ en mediana densidad y PC-E $(2.84 \pm 1.21 \mathrm{~g})$ en baja densidad. El incremento del peso en la fase PC-E continuó en aumento con una leve disminución en la semana
13 de cultivo $(10.70 \pm 1.16 \mathrm{~g})$. Los pesos en la fase PC-PE no tuvieron el mismo nivel de crecimiento siendo inferior al de la fase PC-E en la semana $10(6.11 \pm 1.83 \mathrm{~g}$ y $7.98 \pm$ $0.90 \mathrm{~g}$, respectivamente), cuando se trasladaron a la fase PC-PE-E en baja densidad. En esta fase decayó la curva de crecimiento en la primera semana $(5.48 \pm 0.61 \mathrm{~g})$, pero luego tuvo una leve mejoría alcanzando un peso similar $(12.10 \pm 1.70 \mathrm{~g})$ al de la fase PC-E $(12.14 \pm 1.28 \mathrm{~g})$ dos semanas después. En comparación con la curva teórica de crecimiento de $1.0 \mathrm{~g} / \mathrm{semana}$, las medidas en todas las fases fueron inferiores con una diferencia de 2.9 $\mathrm{g}$ en la PC-E $(12.14 \pm 1.21 \mathrm{~g})$ y $4.3 \mathrm{~g}$

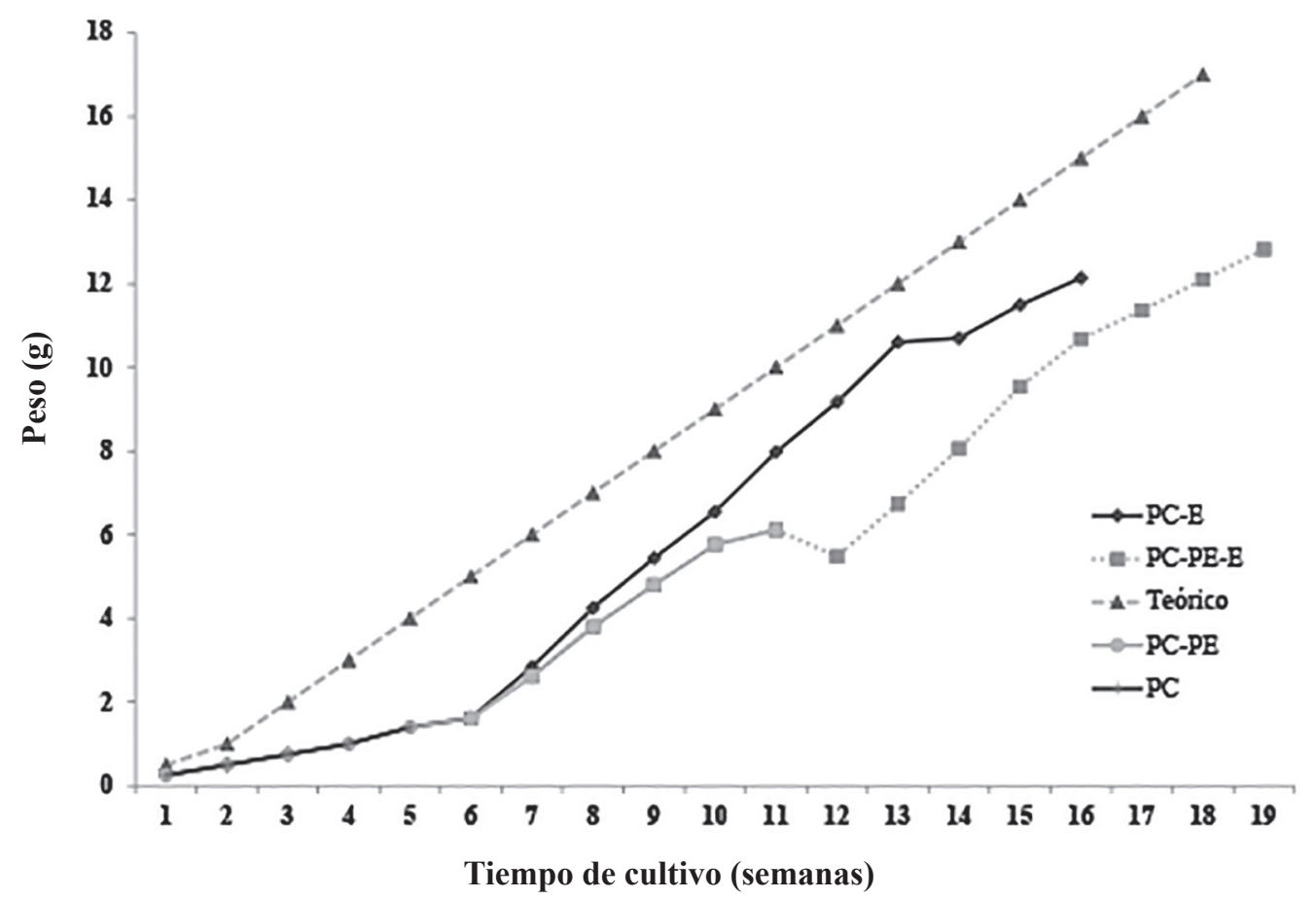

Fig. 1. Incremento en peso de Litopenaeus vannamei en las fases PC y PC-PE, y en dos sistemas de engorde: PC-PE-E y PC-E, en el Golfo de Nicoya, 2014

Fig. 1. Weight increment of Litopenaeus vannamei in the MA and MA-DE phases and in two grow-out systems: MA-DE-G and MA-G, in the Gulf of Nicoya, 2014 
en la PC-PE-E $(10.68 \pm 1.21 \mathrm{~g})$ con respecto a un valor teórico de $15.0 \mathrm{~g}$ en la semana 15 de cultivo.

La TEC medida semanalmente de acuerdo con los pesos en la fase PC-PE y de la PC-PE a la PC-PE-E se muestra en la Figura 2. Se nota un aumento considerable en la TEC (de 2.3 a 7.0$8.5 \% \mathrm{~g} /$ día) durante la primera semana de cultivo después de que se trasladaron los juveniles de la PC en alta densidad a las otras fases en mediana (PC-PE) y baja (PC-E) densidad. No obstante, decae abruptamente en las semanas siguientes en ambas fases. En la fase PC-PE llegó a un valor de $0.63 \%$ g/día en la semana 10 antes de trasladarlos a la fase PC-PE-E. Posteriormente mejoró a $2.58 \%$ g/día las siguientes dos semanas en esta fase, pero volvió a decaer hasta llegar a un valor mínimo de $0.7 \% \mathrm{~g} /$ día en la semana 16 de cultivo. La TEC en la fase PC-E continuó decayendo hasta llegar al valor mínimo de $0.12 \% \mathrm{~g} /$ día en la semana 13. Después mejoró levemente hasta quedar con un valor similar al de la fase PC-PE-E en las últimas semanas de cultivo.

\section{DISCUSIÓN}

Las principales variables de la calidad del agua medidas en los estanques no mostraron mayores diferencias con aquellas reportadas en estudios anteriores (Valverde-Moya \&

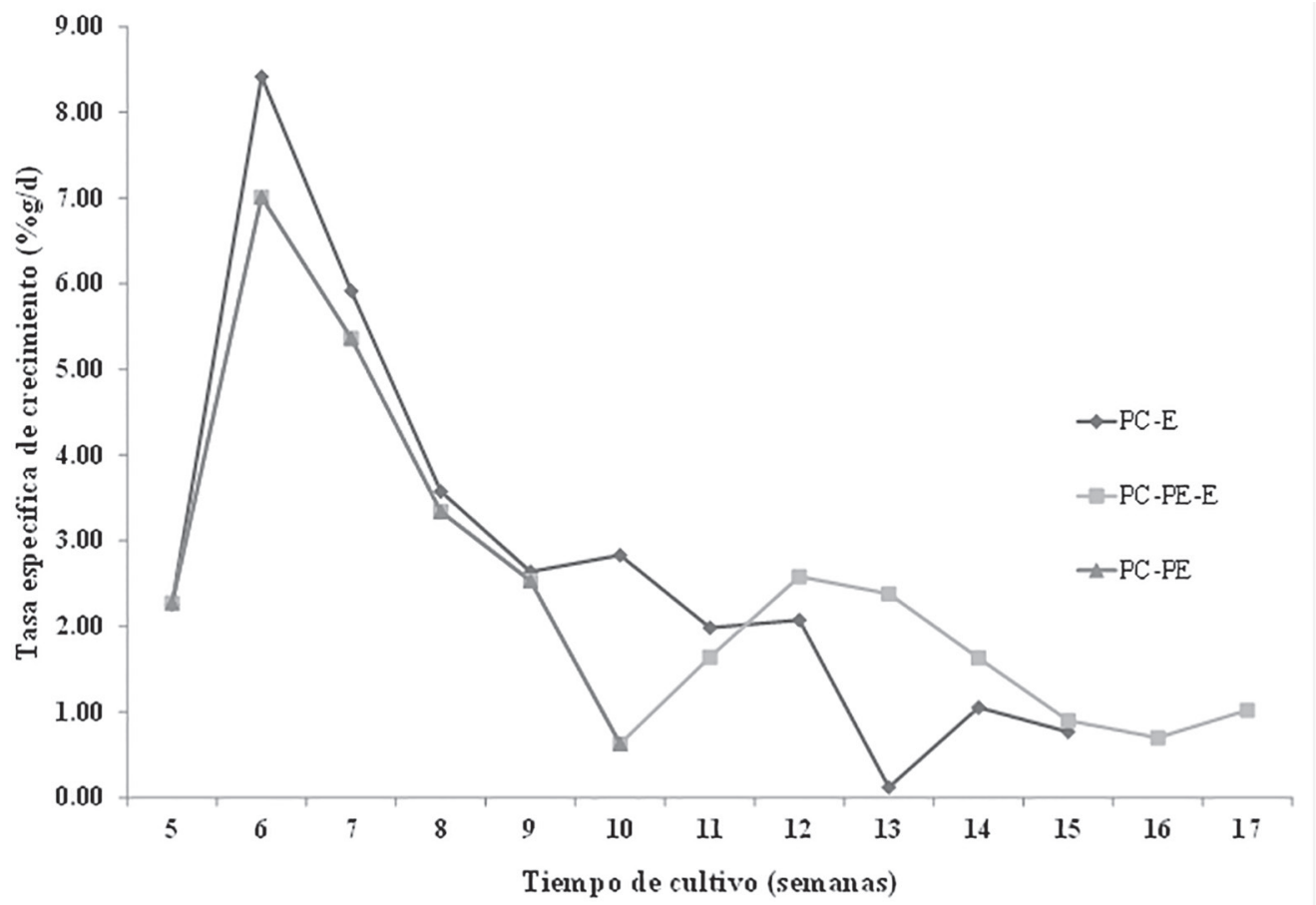

Fig. 2. Tasa específica de crecimiento (\% aumento en peso/d) en las fases PC-PE, PC-E y PC-PE-E, en el Golfo de Nicoya, 2014

Fig. 2. Specific growth rate (\% weight increment/day) in the MA-DE, MA-G and MADE-G phases, in the Gulf of Nicoya, 2014 
Alfaro-Montoya, 2013; 2014) en esta región del país. Los valores estuvieron dentro del rango considerado normal (temperaturas entre 28 y $30^{\circ} \mathrm{C}$, niveles de oxígeno disuelto superiores a $2 \mathrm{mg} / 1$ en las mañanas y una turbidez entre 40 y $45 \mathrm{~cm}$ ) para el cultivo de camarones marinos en las diferentes fases de cultivo en estanques de producción (Boyd, 1989; Scura, 1995; Van Wyk \& Scarpa, 1999; Ferreira et al. 2011). También se dieron los cambios normales de salinidad asociados con la época seca desde diciembre hasta mayo y la época lluviosa desde junio a noviembre (Valverde-Moya \& AlfaroMontoya, 2013).

La mayor sobrevivencia en la PC$\mathrm{PE}$ con respecto a la $\mathrm{PC}$ pudo ser el resultado de la menor densidad de siembra y por haber sembrado juveniles más grandes y mejor adaptados a las condiciones de vida en los estanques. No obstante, la sobrevivencia en la PC puede considerarse muy buena por tratarse de un cultivo en alta densidad que es el parámetro más importante para la efectividad del cultivo (Fóes et al. 2011). Ambas sobrevivencias fueron superiores a las obtenidas en temperaturas similares $\left(65 \%\right.$ a $\left.30^{\circ} \mathrm{C}\right)$ en sistemas superintensivos (100/ $\mathrm{m}^{2}$ ) de biofloc (Hostins et al. 2015) y ligeramente inferiores a la obtenida en un sistema considerado como intensivo con densidades de siembra de 45 camarones $/ \mathrm{m}^{2}$ (83\%; Wyban \& Sweeney, 1989). En otros estudios, no obstante, las sobrevivencias no han sido afectadas por las densidades de siembra (Allan \& Maguire, 1992; Sookying et al. 2011).
Los juveniles de camarón presentaron poco crecimiento en alta densidaden la PC. Este parámetro ha sido una de las mayores limitantes del cultivo en alta densidad en los sistemas de maternidad o precría tanto en camarones marinos (Cervais \& Zeigler, 2013) como en langostinos de agua dulce (Marques \& Lombardi, 2011). En cultivos superintensivos utilizando sistemas de biofloc los crecimientos han sido superiores $(0.36$ $\mathrm{g} /$ semana) a $30^{\circ} \mathrm{C}$ (Hostins et al. 2015). Después el crecimiento mejoró cuando se pasaron a mediana densidad en la PC-PE, pero sin llegar a alcanzar la tasa teórica mínima de un gramo por semana. Resultados similares han sido obtenidos en otros estudios utilizando estas fases de maternidad (PC) y desarrollo (PC-PE) (Komarey et al. 2004).

El valor considerablemente más alto de la TEC en la PC que en la PC$\mathrm{PE}$ es atribuible a la gran diferencia de tamaño entre las poslarvas $(3.3 \mathrm{mg} /$ PL) y los juveniles (1.4 g) sembrados en cada fase a los 35 días de cultivo. La amplia disponibilidad de espacio en el estanque y la abundante proliferación de alimento natural generado y promovido por una adecuada fertilización y maduración también contribuyeron al incremento acelerado de la TEC en las poslarvas (Otoshi et al. 2001). El valor de la TEC en la PC-PE fue de $3.73 \pm 0.66 \% \mathrm{~g} / \mathrm{d}$. El resultado de la producción, donde se combina sobrevivencia y crecimiento, muestra que fue superior en la PC-PE que en la PC. La conversión alimenticia más alta en esta fase parece ser el resultado de la mayor aplicación de alimento $(25.10 \pm 1.21 \mathrm{~kg}$ alim/ha/d). 
Con respecto a las fases de engorde, las sobrevivencias obtenidas cercanas al $85 \%$ pueden considerarse muy buenas y superiores a las obtenidas (76\%) en Venezuela (Krummenauer et al. 2010) con densidades similares de siembra. La similitud del crecimiento en ambas fases de engorde parece mostrar independencia del tamaño inicial sembrado cuando se utiliza una densidad de siembra muy parecida. Este crecimiento fue similar al obtenido en densidades de 20 y $15 / \mathrm{m}^{2}$ con solo fertilización en el estanque (0.68-1.06 $\mathrm{g} /$ semana; Wyban et al. 1987).

Un coeficiente de variación del $15 \%$ en cada fase de engorde indica que en condiciones ambientales estables y de buena calidad del agua, las producciones pueden oscilar entre 850 y $1150 \mathrm{~kg} / \mathrm{ha}$ con un promedio de $1000 \mathrm{~kg} / \mathrm{ha}$ en la PC-E y de 775 a 1050 con un promedio de $900 \mathrm{~kg} /$ ha en la PC-PE-E. Esta similitud en las producciones también parece estar asociada a la densidad de siembra al igual que ocurrió con la sobrevivencia, el crecimiento y el tamaño final alcanzados. Resultados similares en las características de la producción han sido obtenidos al cabo de 16 semanas con la siembra de juveniles de diferentes edades (14 y 21 días de precría) en comparación con poslarvas sembradas directamente en estanques de engorde a densidades de $35 / \mathrm{m}^{2}$ (Zelaya et al. 2007).

El rendimiento de producción medido como la cantidad de kilos por cada 1000 juveniles sembrados también fue muy similar en ambos sistemas de cultivo (PC-E $=10.49$ y PC-PE-E $=10.37$ ) debido a la utilización de densidades parecidas de siembra. Estos valores son ligeramente superiores al obtenido en el sistema de siembra directa de ciclos cortos $(9.2 \mathrm{~kg} /$ millar $)$, pero inferiores al obtenido en el sistema de ciclo largo (14.4 kg/millar) (ValverdeMoya \& Alfaro-Montoya, 2014).

Las diferencias más notorias entre los dos sistemas de engorde parecen hacerse evidentes en la TEC y la conversión alimenticia. La TEC se redujo a menos de la mitad y la conversión alimenticia aumentó considerablemente en la PC-PE-E con respecto a la PC-E. La mayor tasa de crecimiento y la mejor eficiencia en la utilización del alimento en la PC-E pudieron ser el resultado de la ocurrencia de un crecimiento compensatorio parcial en esta fase producto de la drástica disminución en la densidad de siembra al pasar de $85.0 / \mathrm{m}^{2}$ en la PC a $9.4 / \mathrm{m}^{2}$ en esta fase. La influencia del tamaño de siembra (1.4 g en la PC-E y $6.0 \mathrm{~g}$ en la PC-PE-E) también parece mostrar una relación inversamente proporcional con la tasa de crecimiento como ha sido encontrado en langostinos de agua dulce (Marques \& Lombardi, 2011). Una mayor eficiencia en la utilización del alimento y por lo tanto conversiones alimenticias más bajas han sido atribuibles al crecimiento compensatorio en peces (Dobson \& Holmes, 1984).

El crecimiento compensatorio parcial existe cuando los animales no son capaces de alcanzar el mismo tamaño a la misma edad del tratamiento control, a pesar de que muestran un crecimiento más rápido luego de ser resembrados 
(Hostins et al. 2015). Aunque se mejoró la TEC y la conversión alimenticia en la fase PC-E en este estudio, los juveniles no fueron capaces de alcanzar el tamaño de la curva teórica de crecimiento de $1.0 \mathrm{~g} / \mathrm{semana}$ que puede ser considerada como el control. A la semana 15 de cultivo tenían una deficiencia de 2.9 $\mathrm{g}$ con respecto a un valor teórico de 15.0 g. Juveniles en la fase PC-PE-E duraron dos semanas en alcanzar el mismo peso de la PC-E, por lo que se considera la inexistencia de crecimiento compensatorio en esta fase.

La curva de la TEC ha servido para mostrar el crecimiento compensatorio en muchos estudios (Hostins et al. 2015). Nuestros datos parecen indicar que los juveniles de la fase PC venían con una depresión del crecimiento debido a la alta densidad de siembra. Durante los primeros 8 días en las fases siguientes de PC-PE en mediana y PC-E en baja densidad de siembra, los animales lograron mejorar su crecimiento, para luego disminuir en las semanas siguientes. Un nuevo estímulo de crecimiento se detectó durante las primeras dos semanas cuando se pasaron de la fase PC-PE a la PC-PE-E, pero decayó nuevamente. El crecimiento compensatorio, por lo general, es más evidente entre los 10 y 30 días después de que se trasladan los animales acuáticos a condiciones más adecuadas de cultivo (Hostins et al. 2015) y se caracteriza por una mejor utilización del alimento y una mayor eficiencia en la conversión alimenticia, lo que produce una tasa más elevada de crecimiento.
El poco crecimiento de la fase $\mathrm{PC}$ a la PC-PE y de esta fase a la PC-PE-E dio como resultado una mayor duración total del ciclo de cultivo y al final se tradujo en la realización de solo dos ciclos al año. Por tal motivo, la producción en esta fase fue considerablemente inferior. No obstante, estuvo cercana a la obtenida en Texas (1 777 $\mathrm{kg} / \mathrm{ha} / \mathrm{año}$ ) realizando dos ciclos al año bajo condiciones semiintensivas de cultivo (Samocha et al. 2004).

La producción lograda en la fase PC-E es la común cuando se usan densidades de siembras bajas e intermedias en sistemas semiintensivos de cultivo como siguen siendo las comúnmente utilizadas en los países latinoamericanos (TeichertCoddington \& Rodríguez, 1995). Estos sistemas tienen la ventaja de no depender de altos recambios de agua ni sistemas de aireación suplementaria que elevan los costos y aumentan el riesgo de pérdidas económicas en sistemas más intensivos de cultivo (Valderrama \& Engle, 2002).

\section{CONCLUSIONES Y RECOMENDACIONES}

Los resultados de este estudio muestran que en condiciones de cultivo comercial se da la inexistencia de un crecimiento compensatorio de la PC en densidad alta a la PC-PE en densidad media de siembra. Tampoco hubo crecimiento compensatorio de la PC-PE a la PC-PE-E con la densidad baja de siembra de los cultivos tradicionalmente utilizados de manera convencional en el país. Crecimiento compensatorio parcial se registró entre la PC y la PC-E con un cambio radical de densidad de siembra de alta a baja in- 
dependientemente del tamaño inicial de los juveniles.

El cultivo de camarones en alta densidad en la fase de maternidad o precría parece ser una buena estrategia de producción para aumentar la productividad de los cultivos cuando se pasan inmediatamente a la fase de engorde definitivo. Tiene varias ventajas como: las altas sobrevivencias, el aumento en el tamaño de siembra, la reducción de la susceptibilidad del animal a las nuevas condiciones de cultivo y el crecimiento compensatorio parcial. No obstante, los resultados de este estudio deben ser interpretados con precaución porque existen factores como las condiciones climáticas, la calidad del agua y la presencia de enfermedades que varían todos los años y tienen una influencia directa en los rendimientos de producción. Debe plantearse la posibilidad de sustituir la $\mathrm{PC}$ en estanques de tierra por sistemas de tanques bajo techo en forma de invernaderos, con aireación y altas densidades de siembra por menos días (10 a 20), como es la tendencia común en otros países de Latinoamérica.

Con base en los resultados de este estudio es conveniente definir dos tipos de cultivo en fases, adicionales a los ya existentes en el país. Se trata de dos ciclos de tres fases (PC-PE-E) y tres ciclos de dos fases (PC-E) al año. Cada uno tiene sus propias características según el tipo de siembra, manejo, cosechas por año y productividad, por lo que pueden considerarse como sistemas distintos de cultivo.

\section{AGRADECIMIENTOS}

Los autores agradecen la cooperación de los productores en las fincas camaroneras ubicadas en Lepanto, Puntarenas. Un particular agradecimiento a Fernando Vives, biólogo asesor de las fincas estudiadas, y a los evaluadores por sus valiosas observaciones.

\section{BIBLIOGRAFÍA}

Allan, G. \& Maguire, G. (1992). Effects of stocking density on production of Penaeus monodon Fabricius in model farming ponds. Aquaculture, 107(1), 49-66. doi: 10.1016/0044-8486(92)90049-Q.

Boyd, C. (1989). Water quality management and aeration in shrimp farming. Fisheries and Allied Aquaculture Department Series No. 2. Alabama Agricultural Experiment Station, Auburn University. Alabama, EE. UU.: Birmingham Publishing Co.

Cervais, N. \& Zeigler, T. (2013). Feeding considerations for hyperintensive shrimp nursery systems. Global Aquaculture Advocate, 5(18), 50-52. Recuperado en julio 1, 2015, disponible en $\mathrm{http} / / /$ gaalliance.org/advocate-magazine/ past-spanish-issues/

Delgado-Vidal, F., Gallardo-Collí, A., Cuevas-Pérez, L. \& García-Ulloa, M. (2009). Crecimiento compensatorio en tilapia Oreochromis $n i$ loticus posterior a su alimentación con harina de plátano. Av. en Inv. Agropecuaria, 13(2), 55-70.

Dobson, S. \& Holmes, R. (1984). Compensatory growth in the rainbow trout, 
Salmo gairdieri Richarson. J. Fish Biology, 25(6), 649-656. doi: 10.1111/ j.1095-8649.1984.tb04911.x.

Ferreira, N., Boneti, C. \& Seiffert, W. (2011). Hydrological and water quality indices as management tools in marine shrimp culture. Aquaculture, 318(3-4), 425-433. doi: 10.1016/j.aquaculture.2011.05.045.

Fóes, G. K., Fróes, C., Krummenauer, D., Poersch, L. \& Wasielesky, W. (2011). Nursery of Pink Shrimp Farfantepenaeus paulensis in Biofloc Technology Culture System: Survival and Growth at Different Stocking Densities. J. Shellfish Res., 30, 367373. doi: 10.2983/035.030.0224.

Fraga, I., Galindo, J., Arazoza, M., Sánchez, A., Jaime, B. \& Álvarez, S. (2003). Evaluación de niveles de proteína y densidades de siembra en el crecimiento del camarón blanco Litopenaeus schmitti. Rev. Cub. Invest. Pesq., 23(1), 36-39.

Hostins, B., Braga, A., Lopes, D. L. A., Wasielesky, W. \& Poersch, L. H. (2015). Effect of temperature on nursery and compensatory growth of pink shrimp Farfantepenaeus brasilensis reared in a superintensive biofloc system. Aquacult. Eng. (in press). doi: 10.1016/j. aquaeng.2015.03.002.

Komarey, R., Moss, K. \& Shaun, M. (2004). Effects of artificial substrate and stocking density on the nursery production of pacific white shrimp Litopenaeus vannamei. J. World Aquac. Soc., 35(4), 536-542. doi: 10.1111/j.1749-7345.2004.tb00121.x. Krummenauer, D., Cavalli, R. O., Ballester, E. L. C. \& Wasielesky,
W. Jr. (2010). Feasibility of pacific white shrimp Litopenaeus vannamei culture in southern Brazil: effects of stocking density and a single or a double CROP management strategy in earthen ponds. Aquac. Res., 41(2), 240-248. doi: 10.1111/j.1365-2109.2009.02326.x. Marques, H. \& Lombardi, J. (2011). Compensatory growth of Malaysian prawns reared at high densities during the nursery phase. R. Bras. Zootec., 40(4), 701-707. doi: 10.1590/ S1516-35982011000400001.

Nunes, A. (2011). Brazil's intensive shrimp nursery systems: Improve P. L. management, shorten growout. Global Aquaculture Advocate, 14(1), 27-29. Recuperado en julio 1, 2015, disponible en http:// gaalliance.org/advocate-magazine/ past-spanish-issues/

Otoshi, C., Montgomery, D., Look, A. \& Moss, M. (2001). Effects of diet and water source on the nursery production of pacific white shrimp Litopenaeus vannamei. J. World Aquac. Soc., 32(2), 243-249. doi: 10.1111/ j.1749-7345.2001.tb01102.x.

Samocha, T. M., Lopez, I. M., Jones, E. R., Jackson, S. \& Lawrence, A. L. (2004). Characterization of intake and effluent waters from intensive and semiintensive shrimp farms in Texas. Aquac. Res., 35(4), 321-339. doi: 10.1111/j.1365-2109.2004.01002.x. Scura, E. (1995). Dry season production problems on shrimp farms in 
Central America and the Caribean basin. In C. Browdy \& J. Hopkins (Eds.), Swimming through troubled waters (pp. 200-213). Luisiana, EE. UU.: World Aquaculture Society.

Sookying, D., Soller, F., Davis, A. \& Hanson, T. (2011). Effects of stocking density on the performance of Pacific white shrimp Litopenaeus vannamei cultured under pond and outdoor tank conditions using a high soybean meal diet. Aquaculture, 319(2), 232-239. doi: 10.1016/j. aquaculture.2011.06.014.

Stumpf, L., Calvo, N. \& López, L. (2009). Crecimiento compensatorio en juveniles tempranos de la langosta de agua dulce Cherax quadricarinatus (Parastacidae): I. Protocolo de alimentación-inanición. Ponencia presentada en el XII Congreso Nacional de Acuicultura, Fundación Observatorio Español de Acuicultura, Madrid, España. Teichert-Coddington, D. \& Rodriguez, R. (1995). Semi-Intensive commercial grow-out of Penaeus vannamei fed diets containing differing levels of crude protein during wet and dry seasons in Honduras. J. World Aquac. Soc., 26(1), 72-79. doi: 10.1111/ j.1749-7345.1995.tb00211.x.

Valderrama, D. \& Engle, C. (2002). Economic optimization of shrimp farming in Honduras. J. World. Aquac. Soc., 33(4), 398-409. doi: 10.1111/ j.1749-7345.2002.tb00019.x.

Valverde-Moya, J. \& Alfaro-Montoya, J. (2013). La experiencia del cultivo comercial de camarones marinos en estanques de producción en Costa Rica. Rev. Mar. Cost., 5, 87-105.

Valverde-Moya, J. \& Alfaro-Montoya, J. (2014). Productividad y rentabilidad del cultivo de camarones marinos en el Golfo de Nicoya, Costa Rica. Rev. Mar. Cost., 6, 37-53. doi: 10.15359/revmar.6.3.

Van Wyk, P. \& Scarpa, J. (1999). Water Quality Requirements and Management. In P. Van Wyk, M. Davis-Hodgkins, R. Laramore, K. L. Main, J. Mountain \& J. Scarpa (Eds.), Farming Marine Shrimp in Recirculating Freshwater Systems (pp. 128-138). Tallahassee, FL. EE. UU.: Florida Department of Agriculture and Consumers Services.

Wyban, J., Lee, C., Sato, V., Sweeney, J. \& Richards Jr., W. (1987). Effect of stocking density on shrimp growth rates in manure-fertilized ponds. Aquaculture, 61(1), 23-32. doi: 10.1016/0044-8486(87)90334-6.

Wyban, J. \& Sweeney, N. (1989). Intensive shrimp grow out trials in a round pond. Aquaculture, 76(3-4), 215-225. doi: 10.1016/0044-8486(89)90076-8.

Zelaya, O., Rouse, D. \& Davis, A. (2007). Grow out of pacific white shrimp, Litopenaeus vannamei, stocked into production ponds at three different ages. J. World Aquac. Soc., 38(1), 92-101. doi: 10.1111/j.1749-7345.2006.00077.x. 